\section{The future of Clinical Utility Gene Cards in the context of next-generation sequencing diagnostic panels}

European Journal of Human Genetics (2014) 22, 1247; doi:10.1038/ejhg.2014.23; published online 19 February 2014

EuroGentest, an EU-funded Coordination Action, aims to harmonize genetic testing across Europe. EuroGentest unit 2 'Genetic testing as part of health care' and the European Society of Human Genetics promote and coordinate the establishment of 'Clinical Utility Gene Cards' (CUGCs). These guidelines focus on the ability of a genetic test to significantly affect the clinical setting and patient outcome and hereby evaluate the benefits and risks of the test application. CUGCs, mainly aimed at clinicians, geneticists, referrers, service providers and payers in their decision to offer a genetic test to a person, are freely accessible. ${ }^{1,2}$

To establish clinical utility guidelines covering diagnostic nextgeneration sequencing (NGS) approaches, the current disease-specific format of the CUGC guidelines needs to be modified. Most importantly, core sets of genes representing state-of-the-art diagnostic approaches for a given disease (group) need to be defined. In a first step, we have started to build-up an NGS panel data collection, conceptually similar to a proposal of the Dutch Society for Clinical Genetic Laboratory Diagnostics working group. ${ }^{3}$ We have identified 28 laboratories having launched a total of 944 clinical NGS tests covering 2882 genes through an internet search using the web search engine Google. The keywords that were used as search terms were 'next-generation sequencing panel' and 'NGS panel'.

In the panel list we wish to collect comprehensive data from NGS providers including panel name and genes tested; the precise data requested are listed in the file described below. We here link this information to the according disease(s) and genetic background, including OMIM numbers, using data collected from Orphanet and Orphadata, respectively. Although directories of NGS panel providers already exist, ${ }^{4}$ to the best of our knowledge, this will be the first directory of NGS panels specifying the tests they contain. Our main purpose is to give users the opportunity to quickly identify diagnostic options according to different search terms, for example diseases, genes or countries. An overlap of tested genes between different providers can be determined by comparison and genes deemed essential by the providers can be easily identified, serving as the first step in the establishment of CUGCs for NGS-based genetic test applications in diagnostics. EuroGentest currently prepares a guideline about the application of NGS in the diagnostic setting that will be taken into consideration for the adaptation of the CUGC format (Gert Matthijs, personal communication, 20 December 2013).

A prototype of our data collection is available at the EuroGentest website: https://eurogentest.eshg.org/index.php?id=668. We encourage NGS providers to contact us regarding their current services and to include them in this database.

\section{CONFLICT OF INTEREST}

The authors declare no conflict of interest.

\section{ACKNOWLEDGEMENTS}

This work was supported by EuroGentest2 (Unit 2: 'Genetic testing as part of health care'), a Coordination Action under FP7 (Grant Agreement Number 261469) and the European Society of Human Genetics.

\section{Anna Dierking ${ }^{*, 1}$ and Jörg Schmidtke ${ }^{1}$ ${ }^{1}$ Institute of Human Genetics, Hannover Medical School, Hannover, Germany E-mail: eurogentest@mh-hannover.de}

\footnotetext{
1 Dierking A, Schmidtke J, Matthijs G, Cassiman JJ: The EuroGentest Clinical Utility Gene Cards continued. Eur J Hum Genet 2013; 21: 1.

2 Schmidtke J, Cassiman JJ: The EuroGentest clinical utility gene cards. Eur J Hum Genet 2010; 18: 1068.

3 Weiss MM, Van der Zwaag B, Jongbloed JD et al: Best practice guidelines for the use of next-generation sequencing applications in genome diagnostics: a National Collaborative Study of Dutch Genome Diagnostic Laboratories. Hum Mutat 2013; 34: 1313-1321.

4 Rehm HL: Disease-targeted sequencing: a cornerstone in the clinic. Nat Rev Genet 2013; 14: 295-300.
} 